\title{
Review
}

\section{Astroparticle physics with solar neutrinos}

\author{
By Masayuki NAKAHATA ${ }^{* 1, * 2, \dagger}$
}

(Communicated by Toshimitsu YAMAZAKI, M.J.A.)

\begin{abstract}
Solar neutrino experiments observed fluxes smaller than the expectations from the standard solar model. This discrepancy is known as the "solar neutrino problem". Flux measurements by Super-Kamiokande and SNO have demonstrated that the solar neutrino problem is due to neutrino oscillations. Combining the results of all solar neutrino experiments, parameters for solar neutrino oscillations are obtained. Correcting for the effect of neutrino oscillations, the observed neutrino fluxes are consistent with the prediction from the standard solar model. In this article, results of solar neutrino experiments are reviewed with detailed descriptions of what Kamiokande and Super-Kamiokande have contributed to the history of astroparticle physics with solar neutrino measurements.
\end{abstract}

Keywords: neutrino, solar neutrino, neutrino oscillation, sun

\section{Introduction}

In 1938, Hans Bethe predicted that the main energy source of the sun is thermonuclear reactions inside the sun. ${ }^{1)}$ The gravitational potential energy of the sun is too small to explain its long lifetime $(\sim 4.6$ billion years) while nuclear fusion reactions naturally explain its longevity. Copious amounts of electron-type neutrinos $\left(\nu_{\mathrm{e}}\right.$ 's $)$ are predicted to be produced through nuclear fusion reactions and solar neutrino experiments provide direct surveys of deep inside the sun. Because of the limited knowledge of nuclear fusion cross sections at that time, it was not known which reactions (either the pp-chain or the CNO-cycle) were the dominant energy production mechanisms in the sun when Hans Bethe created his theory. R. Davis constructed his chlorine experiment to identify the main fusion reactions in the 1960's. The flux observed by the chlorine experiment was small and Davis concluded that the main fusion reactions were not from the CNO cycle. However, the observed flux was much smaller than the prediction from the standard solar model even if the pp-chain is assumed to be the main process.

*1 Kamioka Observatory, Institute for Cosmic Ray Research, University of Tokyo, Japan.

*2 Institute for the Physics and Mathematics of the Universe, Todai Institute for Advanced Study, University of Tokyo, Japan.

$\dagger$ Correspondence should be addressed: M. Nakahata, Kamioka Observatory, Institute for Cosmic Ray Research University of Tokyo, Higashi-Mozumi, Kamioka-cho, Hida, Gifu 506-1205, Japan (e-mail: nakahata@suketto.icrr.u-tokyo.ac.jp).
The chlorine experiment used a radiochemical method which collected argon atoms produced by neutrino reactions. Because of the unfamiliar technique of the experiment, it was unable to convince people that the solar neutrino problem could be attributed to properties of the neutrino or errors in the standard solar model.

In 1988, the Kamiokande experiment observed solar neutrinos in real time using neutrino-electron scattering. Because the solar neutrino signals were observed as an event excess in the direction of the sun, there was no doubting their origin. The observed solar neutrino flux was about $50 \%$ of the prediction and confirmed the solar neutrino problem. Although the chlorine and Kamiokande experiments showed that the solar neutrino problem was due to something in the solar model or the neutrino itself, it was not able to find the final solution because of large uncertainties in the model predictions.

In the early 1990's, the gallium experiments (SAGE and GALLEX) started to measure low energy solar neutrinos. They also observed a flux smaller than the prediction and the possibility of neutrino oscillations providing the solution to the solar neutrino problem became increasingly probable.

In 1996, Super-Kamiokande, which had 30 times more fiducial volume than that of Kamiokande, started taking data. It detected about 22,400 solar neutrino events by 2001 and the ${ }^{8} \mathrm{~B}$ solar neutrino flux was measured with an accuracy of $3 \%$ using neutrino-electron scattering. In 2001, the SNO group 
announced a ${ }^{8} \mathrm{~B}$ flux measurement using chargedcurrent neutrino-deuteron interactions, and comparisons between the Super-Kamiokande and SNO results gave direct evidence for model-independent solar neutrino oscillations. The evidence was further strengthened by neutral-current measurements from SNO. In 2002, combining the results of the solar neutrino experiments, global analyses showed that the most preferable oscillation parameter is the Large Mixing Angle (LMA; the solution with a mass square difference $\left(m_{2}^{2}-m_{1}^{2}\right)$ of $10^{-5}-10^{-4} \mathrm{eV}^{2}$ and a mixing angle $(\theta)$ of $\left.\sin ^{2}(2 \theta)=0.5-1\right)$. In 2008, the Borexino experiment measured the flux of ${ }^{7} \mathrm{Be}$ solar neutrinos and further confirmed the existence of neutrino oscillations.

In this article, results from solar neutrino experiments are reviewed with detailed descriptions of Kamiokande and Super-Kamiokande. In Section 2, the standard solar model and its neutrino flux predictions are described. The results of the chlorine experiment are described in Section 3 and the observations from Kamiokande are described in Section 4. The high precision measurements of solar neutrinos by Super-Kamiokande are described in Section 5, and results of other experiments are reviewed in Section 6. Based on the results of solar neutrino experiments, solar neutrino oscillation is discussed in Section 7.

\section{Standard solar model}

In the standard solar model (SSM), ${ }^{2)-5)}$ the time evolution of the temperature and pressure at each position in the sun is solved using equations of hydrostatic equilibrium, mass continuity, energy conservation, and energy transport by radiation or convection. The boundary conditions for solving the model are the mass, radius, age, and luminosity of the present sun. Input parameters to the SSM include nuclear fusion cross sections, the initial chemical composition of the sun (elements other than $\mathrm{H}$ and $\mathrm{He}$ ) and the opacity to photons. The SSM assumes that the current surface chemical composition reflects the initial chemical composition, and photo-spectroscopic measurements of the surface are used to estimate its chemical composition.

The SSM predicts that $99 \%$ of the energy production in the sun is due to the $p p$ nuclear reaction chain and the remaining $1 \%$ is due to the $\mathrm{CNO}$ cycle as shown in Fig. 1. The reactions marked by double squares produce neutrinos. The neutrinos from these reactions are referred to as $p p,{ }^{7} \mathrm{Be},{ }^{8} \mathrm{~B}$, $h e p,{ }^{13} \mathrm{~N},{ }^{15} \mathrm{O},{ }^{17} \mathrm{~F}$ neutrinos. The flux of each neutrino

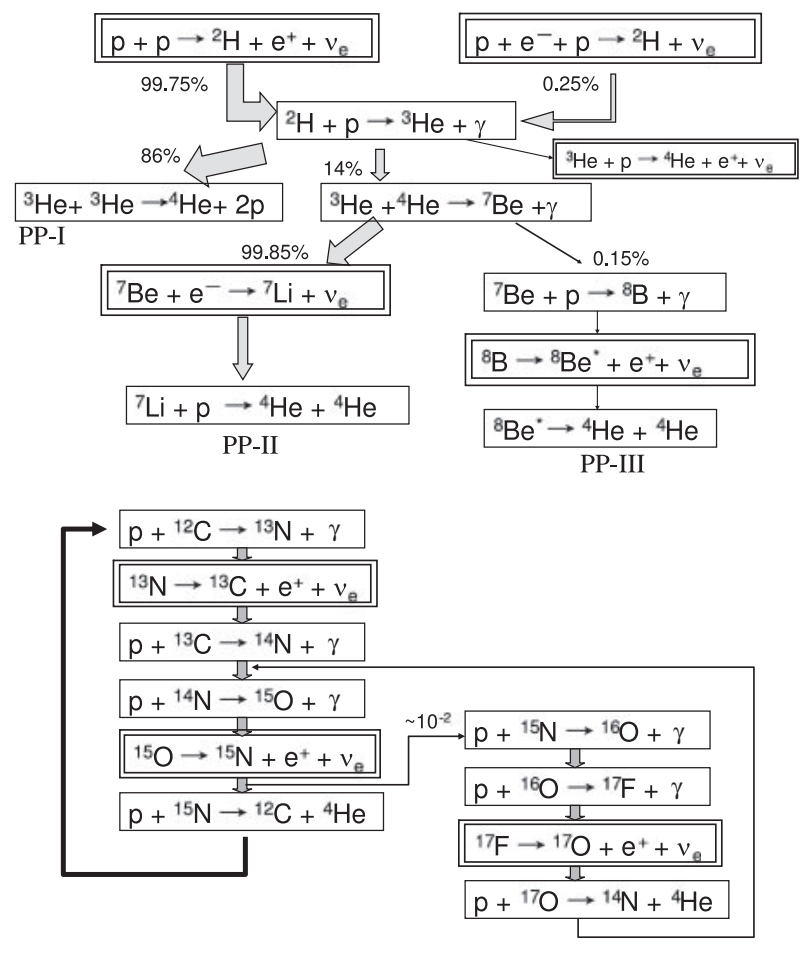

Fig. 1. $p p$ chain and $\mathrm{CNO}$ cycle reactions.

Table 1. Solar neutrino flux predicted by SSM. ${ }^{5)}$ The 2 nd and 3rd columns show the flux predictions using chemical composition from GS98 ${ }^{7)}$ and AGSS09, ${ }^{8)}$ respectively.

\begin{tabular}{cll}
\hline \multirow{2}{*}{ Source } & \multicolumn{2}{c}{ flux $\left(/ \mathrm{cm}^{2} / \mathrm{sec}\right)$} \\
\cline { 2 - 3 } & GS98 & AGSS09 \\
\hline$p p$ & $5.98 \times 10^{10}(1 \pm 0.006)$ & $6.03 \times 10^{10}(1 \pm 0.006)$ \\
${ }^{7} \mathrm{Be}$ & $5.00 \times 10^{9}(1 \pm 0.07)$ & $4.56 \times 10^{9}(1 \pm 0.07)$ \\
$p e p$ & $1.44 \times 10^{8}(1 \pm 0.012)$ & $1.47 \times 10^{8}(1 \pm 0.012)$ \\
${ }^{8} \mathrm{~B}$ & $5.58 \times 10^{6}(1 \pm 0.14)$ & $4.59 \times 10^{6}(1 \pm 0.14)$ \\
$h e p$ & $8.04 \times 10^{3}(1 \pm 0.30)$ & $8.31 \times 10^{3}(1 \pm 0.30)$ \\
${ }^{13} \mathrm{~N}$ & $2.96 \times 10^{8}(1 \pm 0.14)$ & $2.17 \times 10^{8}(1 \pm 0.14)$ \\
${ }^{15} \mathrm{O}$ & $2.23 \times 10^{8}(1 \pm 0.15)$ & $1.56 \times 10^{8}(1 \pm 0.15)$ \\
${ }^{17} \mathrm{~F}$ & $5.52 \times 10^{6}(1 \pm 0.17)$ & $3.40 \times 10^{6}(1 \pm 0.16)$ \\
\hline
\end{tabular}

type from the latest $\mathrm{SSM}^{5)}$ is shown in Table 1, in which revised nuclear cross sections ${ }^{6)}$ are used. The 2nd and 3rd columns in the table show the flux predictions using chemical compositions obtained by GS98 ${ }^{7)}$ and AGSS09, ${ }^{8)}$ respectively. GS98 is based on a 1-dimensional model of the solar atmosphere that was released in 1998. On the other hand, AGSS09, released in 2009, is based on a 3-dimensional model and the most up-to-date atomic and molecular data are used. So, the AGSS09 based solar model should 


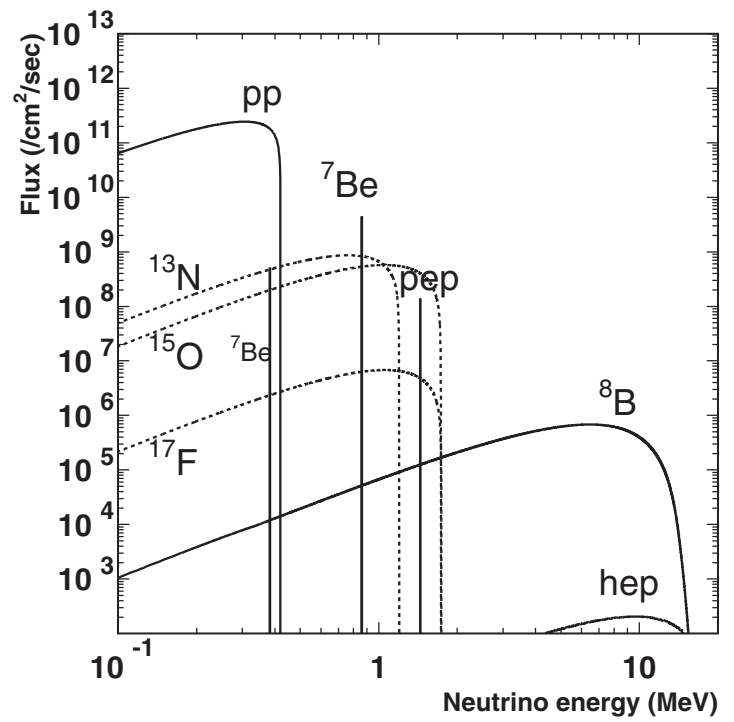

Fig. 2. Solar neutrino energy spectrum expected from the SSM The solid and dashed curves show neutrinos in the $p p$ chain and CNO cycle, respectively. ${ }^{2), 3)}$

be more reliable than the GS98 based solar model. However, the GS98 based solar model can reproduce various observations inside the sun, such as the sound speed profile, depth of the convective zone and the helium abundance, while the AGSS09 based solar model's predictions have large discrepancies with observation. Therefore, the flux predictions of both are shown here.

The energy spectrum of solar neutrinos predicted by the SSM is shown in Fig. 2. The most energetic neutrino is the ${ }^{8} \mathrm{~B}$ neutrino and it was the main neutrino source for the chlorine, Kamiokande, Super-Kamiokande and SNO experiments though its intensity is only about $0.01 \%$ of the total solar flux. The most copious source is the $p p$ neutrino but its maximum energy is only $0.42 \mathrm{MeV}$. The gallium experiments were sensitive to $p p$ neutrinos.

\section{Chlorine experiment}

The chlorine experiment was located in the Homestake gold mine at a depth of 4200 meter water equivalent (m.w.e.). The experiment was started around 1970 and data was taken until 1994. The target for solar neutrinos was ${ }^{37} \mathrm{Cl}$ atoms in 615 tons of $\mathrm{C}_{2} \mathrm{Cl}_{4}$. The neutrino energy threshold of the reaction ${ }^{37} \mathrm{Cl}+\nu_{e} \rightarrow{ }^{37} \mathrm{Ar}+e^{-}$is $0.814 \mathrm{MeV}$ and it is mainly sensitive to ${ }^{8} \mathrm{~B}$ neutrinos. The expected event rate from the $\mathrm{SSM}^{3)}$ was $8.5 \pm 1.8 \mathrm{SNU}$, where one $\mathrm{SNU}$ is $10^{-36}$ captures/atom/sec. The contribution from each neutrino source is $6.6 \mathrm{SNU}$ from ${ }^{8} \mathrm{~B}$

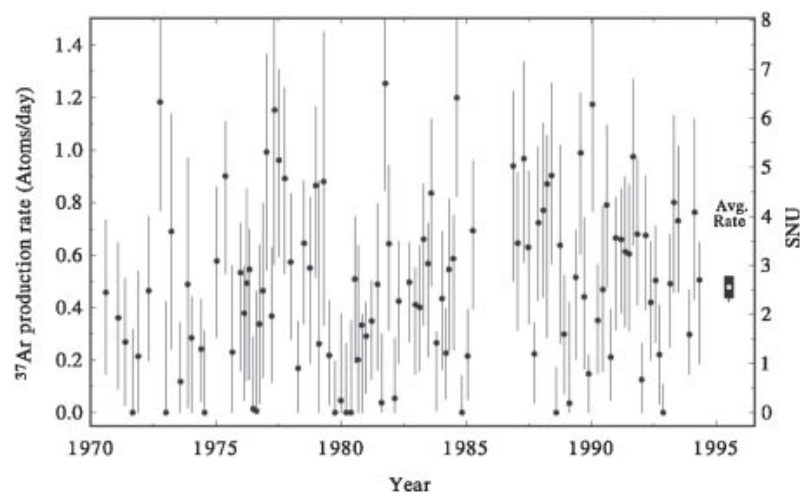

Fig. 3. Data from the Homestake experiment. ${ }^{9)}$ Each data point shows the ${ }^{37} \mathrm{Ar}$ production rate measured in each collection cycle (run). The scale of the right hand side is in SNU. A point shown to the right of the individual data gives the time averaged rate.

neutrinos, 1.2 SNU from ${ }^{7} \mathrm{Be}$ neutrinos, $0.22 \mathrm{SNU}$ from $p e p$ neutrinos, and the remainder from CNO cycle neutrinos. The produced ${ }^{37} \mathrm{Ar}$ atoms are collected once every 60-120 days and the decay of ${ }^{37} \mathrm{Ar}$ was counted using a low background proportional counter. Figure 3 shows the observed production rate of ${ }^{37} \mathrm{Ar}$ in each collection cycle. ${ }^{9)}$ The average event rate observed by the Homestake experiment was

$\phi($ Homestake $)=2.56 \pm 0.16$ (stat. $) \pm 0.16$ (sys.) $\mathrm{SNU}$

The observed event rate was only about $30 \%$ of the SSM prediction and this became the "solar neutrino problem".

\section{Kamiokande}

The Kamiokande detector was constructed in 1983 to search for proton decay. The detector was upgraded for solar neutrino measurements in 1984 and 1985. A schematic view of the Kamiokande detector is shown in Fig. 4. The detector was a 2140 ton water volume viewed by 948 20-inch diameter photomultipliers (PMTs) mounted on a $1 \mathrm{~m}$ grid on the inner surface. The outer (veto) counter, which was constructed during the upgrade in 1984 and 1985 , surrounded the inner detector and was viewed by 123 PMTs. The fiducial volume for the solar neutrino measurement was defined to be the volume $\geq 2 \mathrm{~m}$ from the inner surface and its size was 680 tons. Solar neutrino signals were observed by the Cherenkov radiation of recoil electrons from neutrino-electron scattering. Because of the higher energy threshold for Cherenkov radiation, the Kamiokande detector was sensitive only to ${ }^{8} \mathrm{~B}$ neutrinos. The 


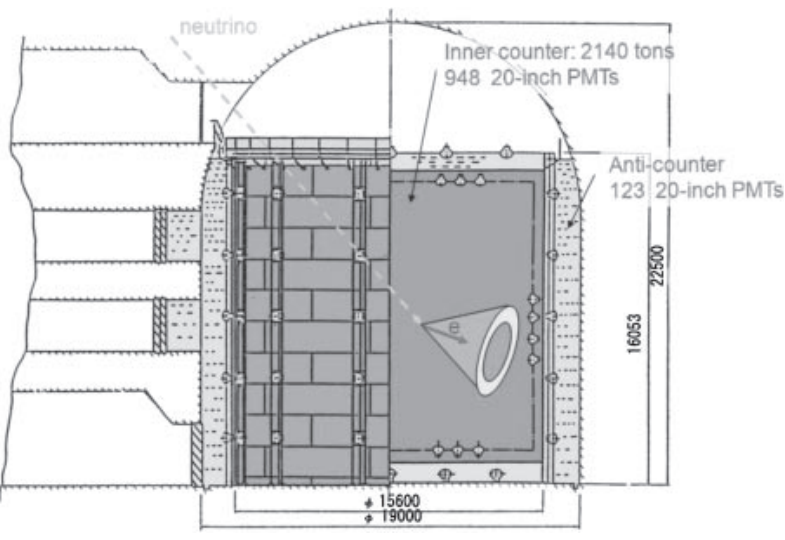

Fig. 4. Schematic view of the Kamiokande detector.

main background source was radon in the water (Cherenkov radiation is produced by the beta decay of ${ }^{214} \mathrm{Bi}$ ), and the detector improvements to remove this radon were performed in 1986. Another background source was spallation products produced by cosmic ray muons. Those events were rejected by applying cuts on the correlation in space and time with cosmic ray muon events. Figure 5 shows the angular distribution to the sun obtained by the initial 450 day data taken from January 1987 to May 1988. ${ }^{10)}$ A clear excess of events was observed in the direction of the sun but the observed rate was about $50 \%$ of the prediction from the SSM (solid histogram in the figure). This observation confirmed the solar neutrino problem and demonstrated that its origin is either the solar model or a property of the neutrino, such as neutrino oscillations.

The Kamiokande detector observed $\sim 600$ solar neutrino events by February 1995 ${ }^{11)}$ and the obtained flux of ${ }^{8} \mathrm{~B}$ neutrino was

$$
\begin{aligned}
& \phi(\text { Kamiokande }) \\
& \quad=2.80 \pm 0.19 \text { (stat.) } \pm 0.33 \text { (sys. }) \times 10^{6} / \mathrm{cm}^{2} / \text { sec. }
\end{aligned}
$$

The observed flux was $48 \pm 3$ (stat.) \pm 6 (sys.) $\%$ of the prediction from the SSM. The energy spectrum of recoil electrons normalized by the predicted spectrum is shown in Fig. 6. The spectrum shape was consistent with a flat spectrum and further discussion with respect to neutrino oscillation was handed to the high statistics measurement by the SuperKamiokande.

\section{Super-Kamiokande}

The Super-Kamiokande (SK) detector is a 50,000 ton water Cherenkov detector with its inner active volume (32,000 tons) viewed by 11,146 $50 \mathrm{~cm}$
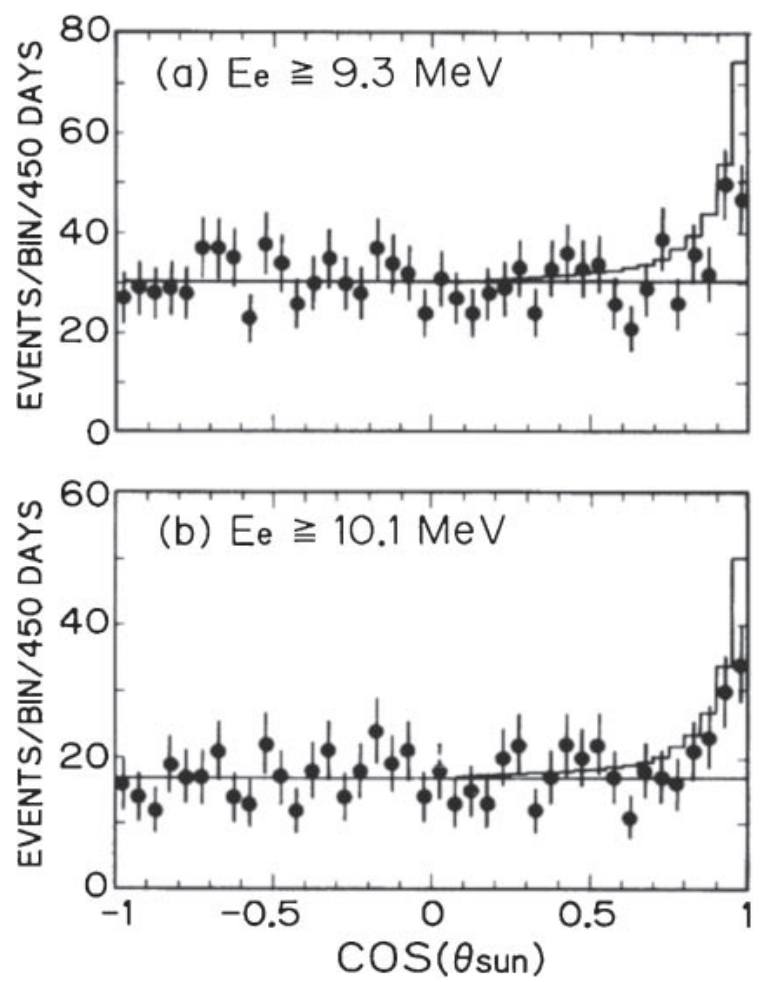

Fig. 5. Angular distribution to the direction of the sun in Kamiokande. ${ }^{10)}$ The plot shows data from the first 450 days of data, taken from January 1987 to May 1988. The solid histogram shows the prediction from the SSM.

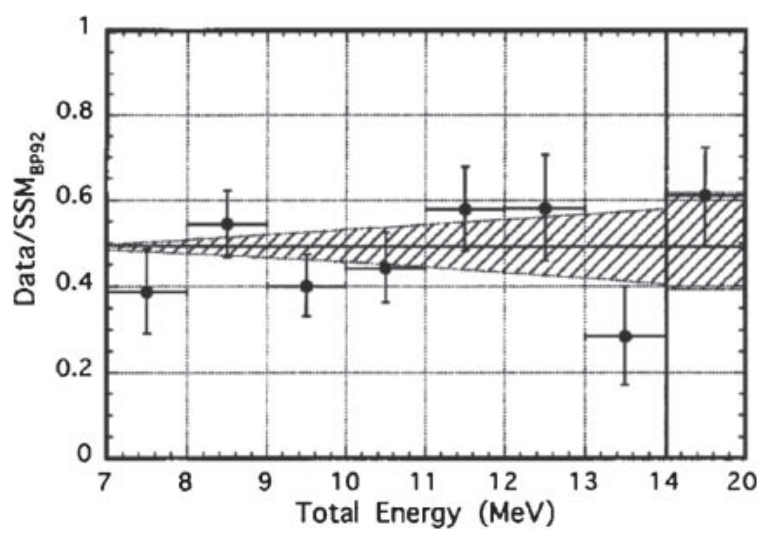

Fig. 6. Energy spectrum of recoil electrons normalized by the predicted spectrum from the 2079 day data set of Kamiokande. The hatched area shows the range of systematic uncertainty.

diameter photomultipliers (PMTs). The fiducial volume for the solar neutrino measurement is defined by the volume more than $2 \mathrm{~m}$ from the surface of the PMTs and has a volume of 22,500 tons. A schematic view of the SK detector is shown in Fig. 7. SK has measured ${ }^{8} \mathrm{~B}$ neutrinos using neutrino-electron scat- 


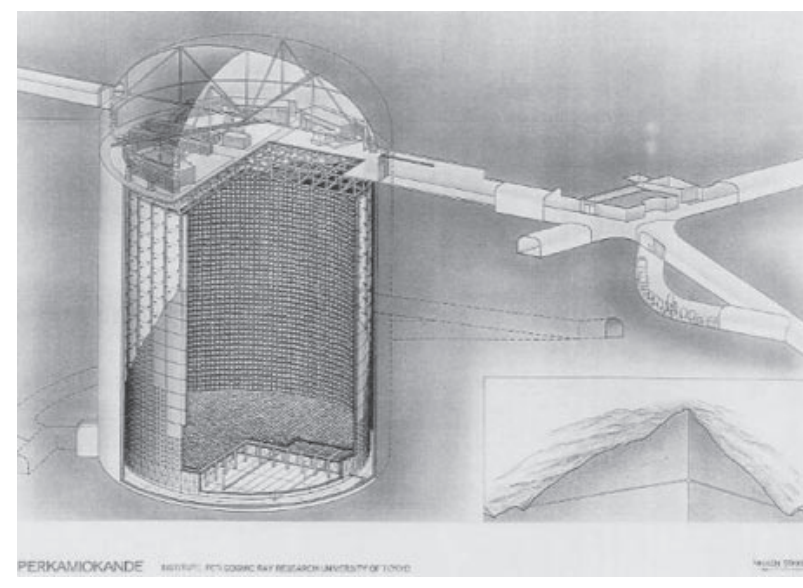

Fig. 7. Schematic view of the Super-Kamiokande detector.

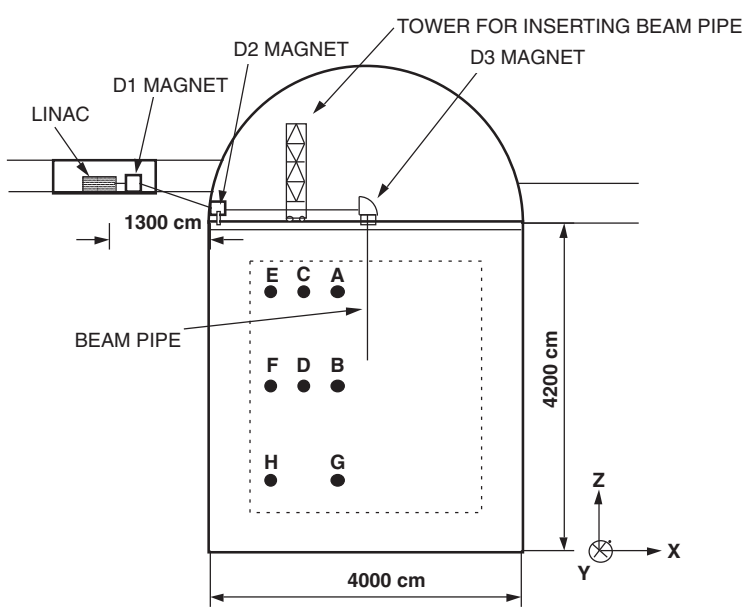

Fig. 8. Schematic view of the electron LINAC calibration system in SK.

tering in the same manner as Kamiokande. The main difference between Super-Kamiokande and Kamiokande is the larger fiducial volume $(30 \times)$ and increased fraction of photo-sensitive coverage $(2 \times)$, which enabled the energy threshold to be lowered to $5 \mathrm{MeV}$ total energy.

In order to measure the ${ }^{8} \mathrm{~B}$ solar neutrino flux its energy spectrum with high precision, special caution was taken in SK. The absolute energy of the detector was calibrated using an electron linear accelerator $(\text { LINAC })^{12)}$ installed at the detector site as show in Fig. 8. The LINAC system was able to generate mono-energetic electrons and inject them at various positions in the detector. A typical energy distribution of LINAC data and its Monte Carlo simulation is shown in Fig. 9. The LINAC system gave a very precise energy scale calibration but it is only accurate
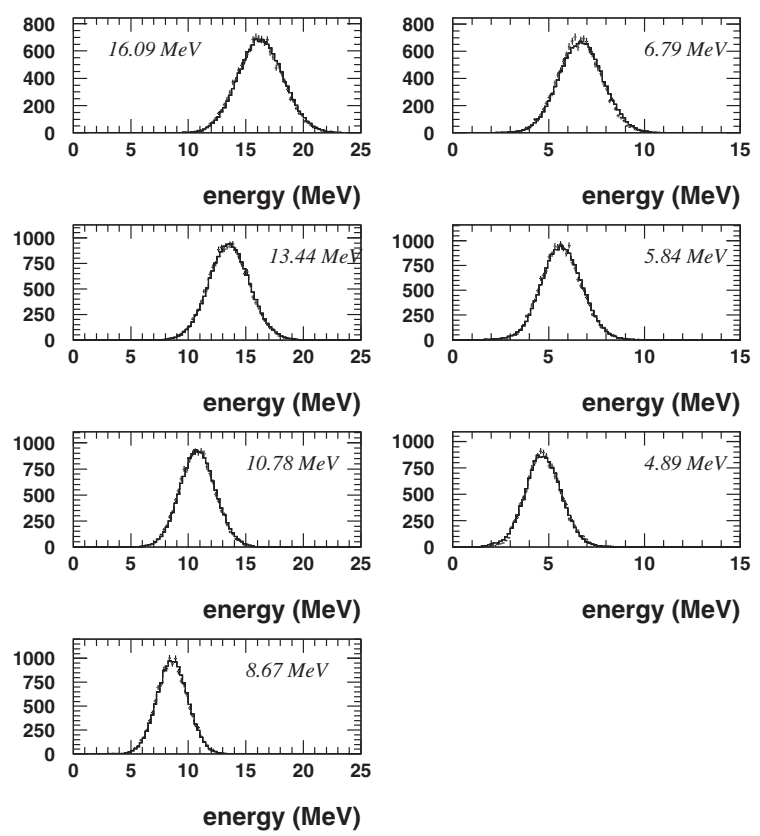

Fig. 9. Energy spectrum of LINAC calibration data (crosses) compared with its MC simulation (histogram).

for vertical downward-going events. In order to calibrate the angular dependence of the energy scale, a ${ }^{16} \mathrm{~N}$ radioactive source ${ }^{13)}$ was used. ${ }^{16} \mathrm{~N}$ 's are produced by fast neutron capture on oxygen nuclei in water. Neutrons were generated by a commercially built Deuteron-Tritium generator which produces $10^{6} 14.2 \mathrm{MeV}$ neutrons per pulse. The main decay mode of ${ }^{16} \mathrm{~N}$ is an electron with a $4.3 \mathrm{MeV}$ maximum energy coincident with a $6.1 \mathrm{MeV}$ gamma ray. A setup of the DT generator is deployed in the SK tank, and it is pulled up by about $2 \mathrm{~m}$ after it emits neutrons in order to avoid shadowing of the Cherenkov light. Because of the precise energy calibration from the LINAC and DT systems, the absolute energy scale of the SK detector is calibrated with an accuracy of $0.64 \%$ (rms). This improved to $0.53 \%$ in the third phase of the SK detector.

The first phase of SK (SK-I) took 1496 days of data from May 1996 to July 2001. ${ }^{14), 15), 16)}$ The second phase (SK-II) took data for 791 days from December 2002 to October 2005 using 5,182 $50 \mathrm{~cm} \mathrm{PMTs.}{ }^{17)}$ The reduced PMT coverage in SK-II ( $50 \%)$ compared to SK-I was due to an accident in November 2001. The detector was fully reconstructed from October 2005 to July 2006 and the third phase (SK-III) ran for 548 days from October 2006 to August 2008. ${ }^{18)}$ The readout electronics were replaced in September 2008 and the fourth phase (SK- 


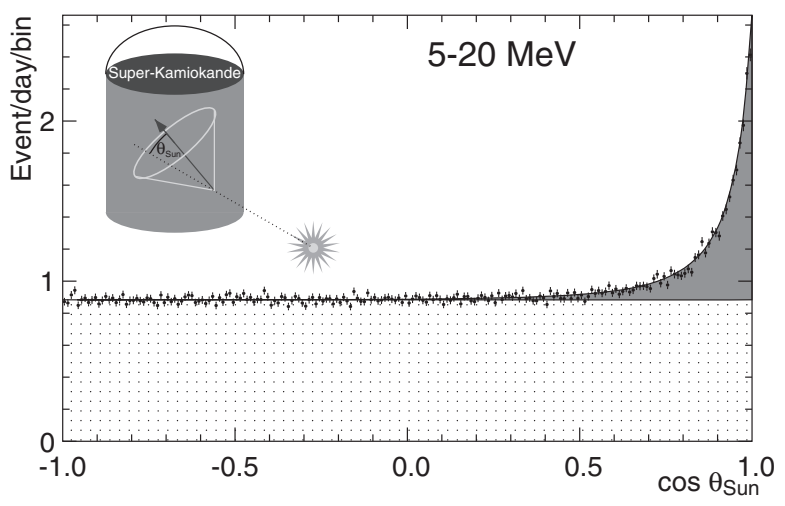

Fig. 10. Angular distribution with respect to the direction of the sun in Super-Kamiokande phase I (from May 1996 to July 2001). ${ }^{16)}$

IV) has been running since then. The energy threshold of the data analyses are $5.0 \mathrm{MeV}$ total energy in SK-I and SK-III, and $7 \mathrm{MeV}$ in SK-II.

Figure 10 shows the angular distribution of solar event candidates with respect to the direction of the sun from SK-I. Solar neutrino events are clearly seen above the flat background distribution. The total number of observed solar neutrino events in SK-I was 22,400 \pm 230 events, which corresponds to 14.5 events/day.

The ${ }^{8} \mathrm{~B}$ solar neutrino fluxes obtained assuming the SSM energy spectrum are

$$
\begin{aligned}
\phi_{S K-I}^{E S}= & 2.35 \pm 0.02 \text { (stat.) } \pm 0.08 \text { (sys.) } \\
& \times 10^{6} / \mathrm{cm}^{2} / \mathrm{sec} \\
\phi_{S K-I I}^{E S}= & 2.38 \pm 0.05(\text { stat. })_{-0.15}^{+0.16}(\text { sys. }) \\
& \times 10^{6} / \mathrm{cm}^{2} / \mathrm{sec} \\
\phi_{S K-I I I}^{E S}= & 2.32 \pm 0.04 \text { (stat.) } \pm 0.05 \text { (sys.) } \\
& \times 10^{6} / \mathrm{cm}^{2} / \text { sec. }
\end{aligned}
$$

The suffix ES denotes the flux measurement performed using neutrino-electron scattering (ES). The results of these three phases agree with each other and the systematic error of SK-III is improved over SK-I because of better control of the energy calibration and more stable water quality. The observed ES flux is 40-50\% of the expectation from the SSM, shown in Table 1.

The energy spectrum shape is important for the discussion of neutrino oscillations. Figure 11 shows the energy spectrum of solar neutrino signals observed in SK-I compared with the expectation from the SSM. ${ }^{3)}$ In order to discuss the shape of the energy spectrum, the bin by bin ratio of Fig. 11 was taken and is shown in Fig. 12. The observed

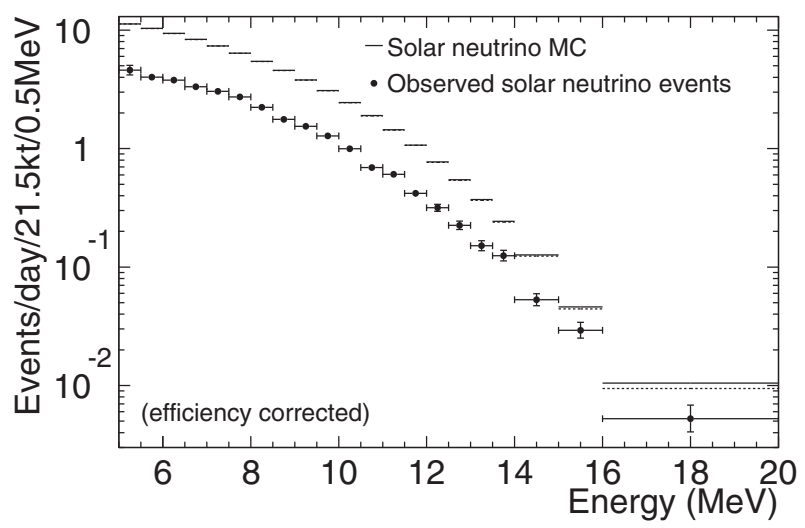

Fig. 11. Energy spectrum of the solar neutrino signal observed in SK-I (data points) and the expectation from the SSM. ${ }^{3)}$

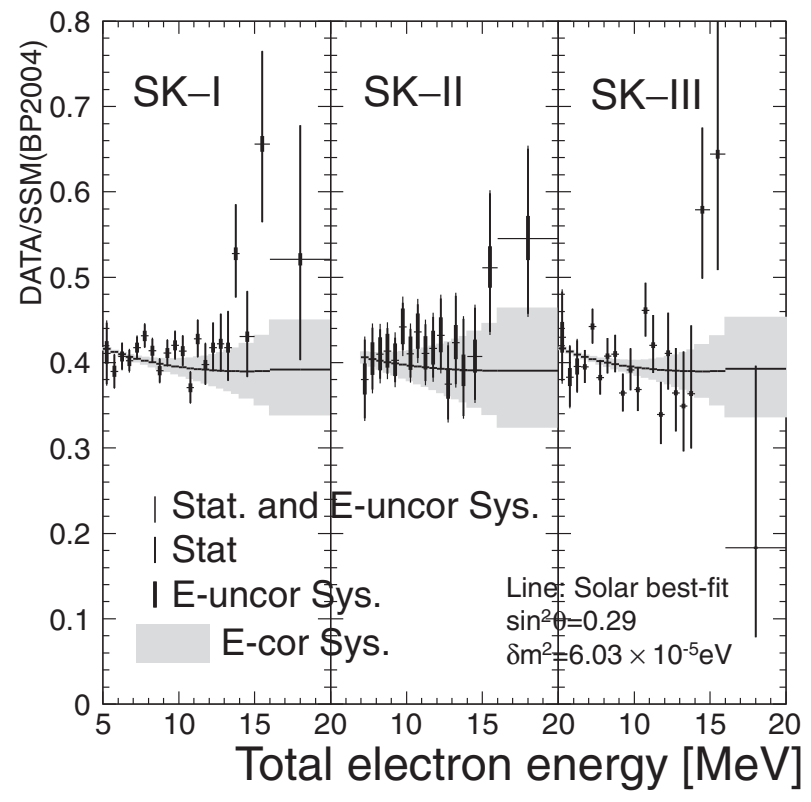

Fig. 12. Measured energy spectrum of SK. The horizontal axis is the visible energy of recoil electrons and the vertical axis shows the flux normalized by the SSM prediction.

energy spectrum shape is consistent with flat. As shown in the figure, in order to observe distortion of the energy spectrum more statistics are needed. This is the main goal of the solar neutrino measurement in SK-IV.

\section{Other solar neutrino experiments}

A. SAGE and GALLEX/GNO. SAGE and GALLEX/GNO were radiochemical experiments using gallium targets conducted since the early 1990s. The SAGE experiment ${ }^{19)-21)}$ has been conducted at the Baksan Observatory, and the GALLEX experi- 

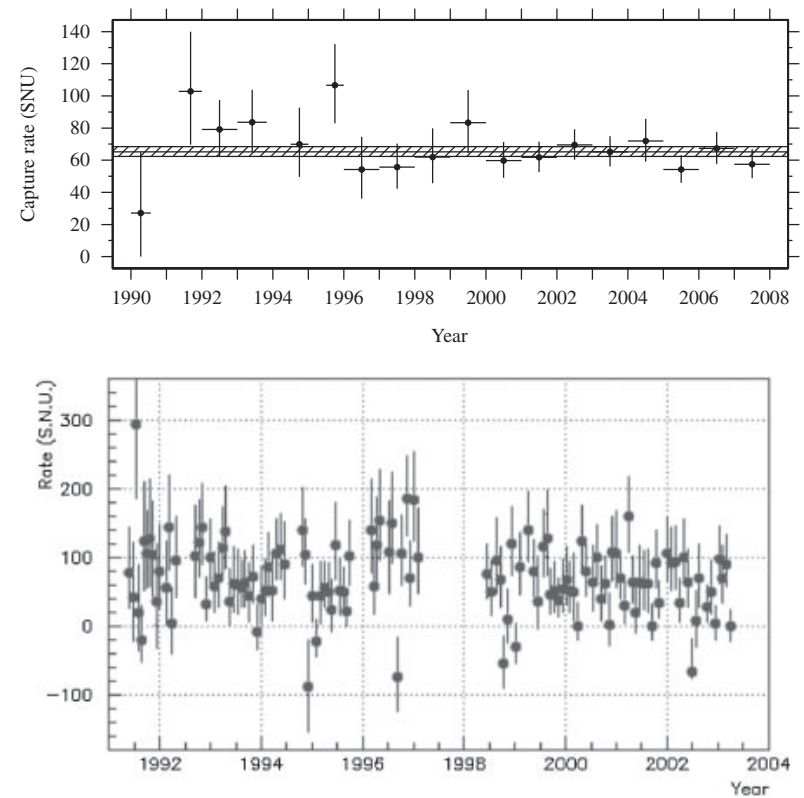

Fig. 13. Upper: yearly average ${ }^{71} \mathrm{Ge}$ production rate of SAGE data from ref. 21. Shaded band shows combined best fit and its uncertainty for all years. Lower: ${ }^{71} \mathrm{Ge}$ production rate of each run in GALLEX/GNO. ${ }^{25)}$

ment ${ }^{22)-24)}$ (later changed to $\mathrm{GNO}^{25)}$ ) at the Gran Sasso Laboratory. The energy threshold of the gallium reaction $\left({ }^{71} \mathrm{Ga}+\nu_{e} \rightarrow{ }^{71} \mathrm{Ge}+e^{-}\right)$is 0.233 $\mathrm{MeV}$ and is mainly sensitive to low energy solar neutrinos. The expected event rate from the $\mathrm{SSM}^{3)}$ is $131_{-10}^{+12}$ SNU and the contribution from each neutrino source is 69.6 SNU from $p p$ neutrinos, $34.8 \mathrm{SNU}$ from ${ }^{7} \mathrm{Be}$ neutrinos, 13.9 $\mathrm{SNU}$ from ${ }^{8} \mathrm{~B}$ neutrinos, 2.9 SNU from pep neutrinos, and the remainder from CNO cycle neutrinos. SAGE used 50 tons of gallium in metallic form and GALLEX/GNO used 30 tons of gallium in a $\mathrm{GaCl}_{3} \cdot \mathrm{HCl}$ solution. The lifetime of ${ }^{71} \mathrm{Ge}$ is 16.5 days and a typical exposure time for one run was 28 days. Figure 13 shows the observed production rate of ${ }^{71} \mathrm{Ge}$ in $\mathrm{SAGE}$ and GALLEX/GNO. The average event rates observed by SAGE and GALLEX/GNO are

$$
\begin{aligned}
\phi(S A G E) & \left.=65.4_{-3.0}^{+3.1} \text { (stat. }\right)_{-2.8}^{+2.6} \text { (sys.) } \mathrm{SNU} \\
\phi(G A L L E X) & \left.=73.1_{-6.0}^{+6.1} \text { (stat. }\right)_{-4.1}^{+3.7} \text { (sys.) } \mathrm{SNU} \\
\phi(G N O) & \left.=62.9_{-5.3}^{+5.5} \text { (stat. }\right)_{-2.5}^{+2.5} \text { (sys.) SNU. }
\end{aligned}
$$

Combining these results, ${ }^{21)}$ the flux measured by the gallium experiments is

$$
\phi(\text { gallium })=66.1 \pm 3.1 \mathrm{SNU}
$$

The observed flux is $50 \%$ of the expectation from the SSM. $^{3)}$
B. SNO. The Sudbury Neutrino Observatory (SNO) detector was a 1000 ton heavy water $\left(\mathrm{D}_{2} \mathrm{O}\right)$ Cherenkov detector located in Sudbury, Canada. Its $945620 \mathrm{~cm}$ diameter PMTs viewed heavy water contained in an acrylic vessel. The SNO detector could measure the $\nu_{e}$ flux from ${ }^{8} \mathrm{~B}$ neutrinos and the flux of all active neutrino flavors through the following interactions:

$$
\begin{array}{ll}
\nu_{e}+d \rightarrow p+p+e^{-} & \text {(charged current (CC)) } \\
\nu_{x}+d \rightarrow p+n+\nu_{x} & \text { (neutral current (NC)) } \\
\nu_{x}+e^{-} \rightarrow \nu_{x}+e^{-} & (\text {electron scattering (ES)) }
\end{array}
$$

where $\nu_{x}$ is any of $\nu_{e}, \nu_{\mu}$ or $\nu_{\tau}$. The first phase of the SNO data (SNO-I) was taken using a pure $\mathrm{D}_{2} \mathrm{O}$ target over 306 days from November 1999 to May $2001{ }^{26)}$ The free neutron from the $\mathrm{NC}$ interaction thermalizes and a $6.25 \mathrm{MeV} \gamma$ ray is emitted following its capture on a deuteron. The capture efficiency is about $30 \%$. The measured fluxes are

$$
\begin{aligned}
\phi_{S N O-I}^{C C}= & 1.76 \pm 0.05 \text { (stat.) } \pm 0.09 \text { (sys.) } \\
& \times 10^{6} / \mathrm{cm}^{2} / \mathrm{sec} \\
\phi_{S N O-I}^{E S}= & 2.39_{-0.23}^{+0.24}(\text { stat. }) \pm 0.12 \text { (sys.) } \\
& \times 10^{6} / \mathrm{cm}^{2} / \mathrm{sec} \\
\phi_{S N O-I}^{N C}= & 5.09_{-0.43}^{+0.44}(\text { stat. })_{-0.43}^{+0.46} \text { (sys.) } \\
& \times 10^{6} / \mathrm{cm}^{2} / \text { sec. }
\end{aligned}
$$

In the second phase of the SNO experiment (SNOII), 2 tons of $\mathrm{NaCl}$ were added to the $\mathrm{D}_{2} \mathrm{O}$ target in order to enhance the detection efficiency of the $\mathrm{NC}$ channel. ${ }^{27)}$ The thermalized neutron was captured by a ${ }^{35} \mathrm{Cl}$ nucleus, which resulted in the emission of a $\gamma$ ray cascade with a total energy of $8.6 \mathrm{MeV}$. The CC and the NC signals were statistically separated using the isotropy of the Cherenkov light pattern and the event's angle to the sun. For example, Fig. 14 shows this angular distribution. The forward peaked signal is due to $\mathrm{ES}$ and backward distribution is due to $\mathrm{CC}$ interactions. $\mathrm{NC}$ events are isotropic with respect to the solar direction. The measured fluxes in SNO-II are

$\phi_{\text {SNO-II }}^{C C}=1.68 \pm 0.06$ (stat. $)_{-0.09}^{+0.08}$ (sys. $) \times 10^{6} / \mathrm{cm}^{2} / \mathrm{sec}$ $\phi_{\text {SNO-II }}^{E S}=2.35 \pm 0.22$ (stat. $)_{-0.15}^{+0.15}$ (sys.) $\times 10^{6} / \mathrm{cm}^{2} / \mathrm{sec}$ $\phi_{\text {SNO-II }}^{N C}=4.94 \pm 0.21$ (stat. $)_{-0.34}^{+0.38}($ sys. $) \times 10^{6} / \mathrm{cm}^{2} /$ sec.

In the third phase of SNO (SNO-III), ${ }^{3} \mathrm{He}$ proportional counters were deployed in the heavy water and the $\mathrm{NC}$ events were measured independently. ${ }^{28)}$ The NC flux measured by SNO-III was

$$
\left.\phi_{S N O-I I I}^{N C}=5.54_{-0.31}^{+0.33} \text { (stat. }\right)_{-0.34}^{+0.36}(\text { sys. }) \times 10^{6} / \mathrm{cm}^{2} / \mathrm{sec} .
$$




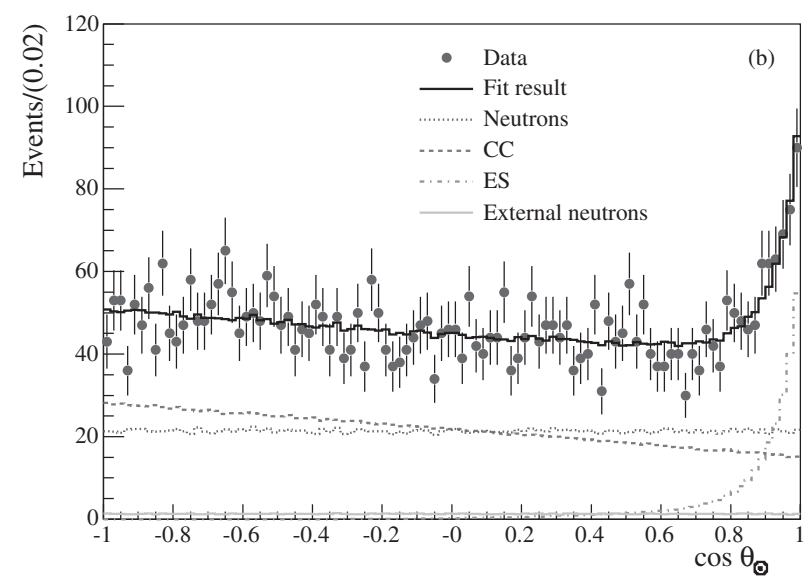

Fig. 14. Angular distribution with respect to the direction of the sun in the SNO salt phase data. ${ }^{27)}$

The energy threshold of the analyses described above are $5.0 \mathrm{MeV}$ and $5.5 \mathrm{MeV}$ kinetic energies for SNO-I and SNO-II, respectively. The SNO group reanalyzed SNO-I and SNO-II data with an effective kinetic energy threshold of $3.5 \mathrm{MeV}$ (LETA analy$\left.\operatorname{sis}^{29)}\right)$. The obtained NC flux by LETA was

$$
\begin{aligned}
\phi_{\text {SNO-LETA }}^{N C}= & \left.5.140_{-0.158}^{+0.160} \text { (stat. }\right)_{-0.117}^{+0.132} \text { (sys.) } \\
& \times 10^{6} / \mathrm{cm}^{2} / \text { sec. }
\end{aligned}
$$

C. Borexino. Borexino is a liquid scintillator detector with an active mass of 278 tons of pseudocumene. Scintillation light is detected via 2212 8-inch PMTs uniformly distributed on the inner surface of the detector. Because of the high light yield of liquid scintillator compared with Cherenkov light, Borexino is sensitive to sub-MeV solar neutrinos. Its first ${ }^{7} \mathrm{Be}$ solar neutrino measurement was reported in ref. 30 based on 192 days of data taken from May 2007 to April 2008. The $0.862 \mathrm{MeV}$ monoenergetic ${ }^{7} \mathrm{Be}$ neutrinos were detected by neutrino-electron scattering. Figure 15 shows the energy spectrum of observed events and it was deconvoluted using the expected shape of the recoil electrons and possible background sources. The extracted ${ }^{7} \mathrm{Be}$ neutrino event rate was

$$
49 \pm 3 \text { (stat.) } \pm 4 \text { (sys.) counts } /(\text { day } \cdot 100 \text { ton }),
$$

while the expected event rate from the SSM was $74 \pm 4$ counts/(day.100 ton).

\section{Summary of experimental results}

Experimental results discussed in the previous sections are summarized in Fig. 16. In this figure, the measured fluxes are compared with the SSM expectation. ${ }^{4}$ Each neutrino source is shown by different color. With the exception of the SNO NC flux, all

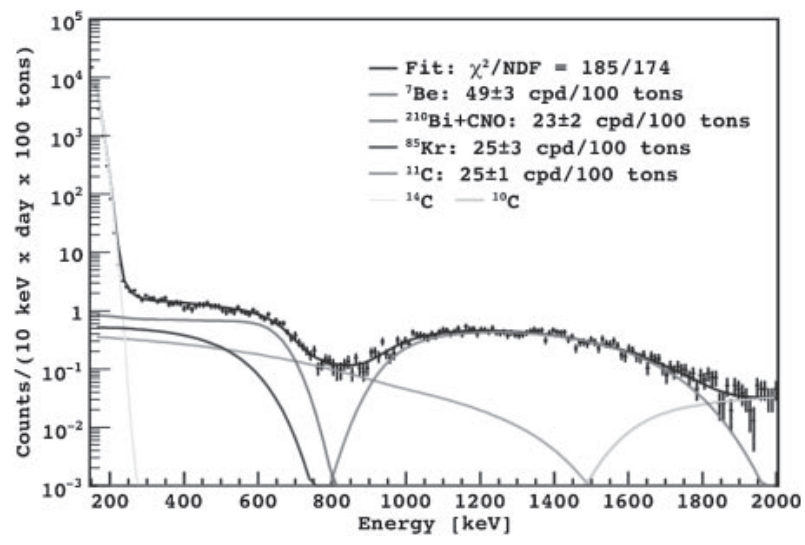

Fig. 15. Energy spectrum from 192 days Borexino data from ref. 30 .

of the observed solar neutrino fluxes are significantly lower than the SSM prediction.

The solution of the deficit of solar neutrinos is discussed in the next section.

\section{Solar neutrino oscillations}

Precise solar neutrino measurements over the last 10 years have shown that the solar neutrino problem is due to neutrino oscillations. In this section, details of solar neutrino oscillations are discussed.

A. Formula for solar neutrino oscillations. The relation between the mass eigenstates of two neutrinos $\left(\nu_{1}\right.$ and $\left.\nu_{2}\right)$ and their interaction eigenstates $\left(\nu_{e}\right.$ and $\left.\nu_{X}(X=\mu, \tau)\right)$ is expressed as

$$
\left(\begin{array}{c}
\nu_{e} \\
\nu_{X}
\end{array}\right)=\left(\begin{array}{cc}
\cos \theta & \sin \theta \\
-\sin \theta & \cos \theta
\end{array}\right)\left(\begin{array}{l}
\nu_{1} \\
\nu_{2}
\end{array}\right)
$$

where $\theta$ is the mixing angle. Solving the time evolution of the neutrino wave function, the probability that produced as electron-type are observed as eletron-type is

$P\left(\nu_{e} \rightarrow \nu_{e}\right)=1-\sin ^{2} 2 \theta \times \sin ^{2}\left(1.27 \times \Delta m^{2} \frac{L}{E}\right)$,

where $\Delta m^{2}$ is the mass squared difference $\left(m_{2}^{2}-m_{1}^{2}\right)$ in units of $\mathrm{eV}^{2}, L$ is the neutrino travel length in meters, and $E$ is neutrino energy in $\mathrm{MeV}$. If the argument of the last sine function, $1.27 \times \Delta m^{2} \frac{L}{E}$, is much larger than $2 \pi$, it averages out and the survival probability becomes:

$$
P\left(\nu_{e} \rightarrow \nu_{e}\right)=1-\frac{1}{2} \sin ^{2} 2 \theta
$$

However, the effect of matter in the sun and the earth must be considered. ${ }^{31), 32)}$ The Schrödinger 


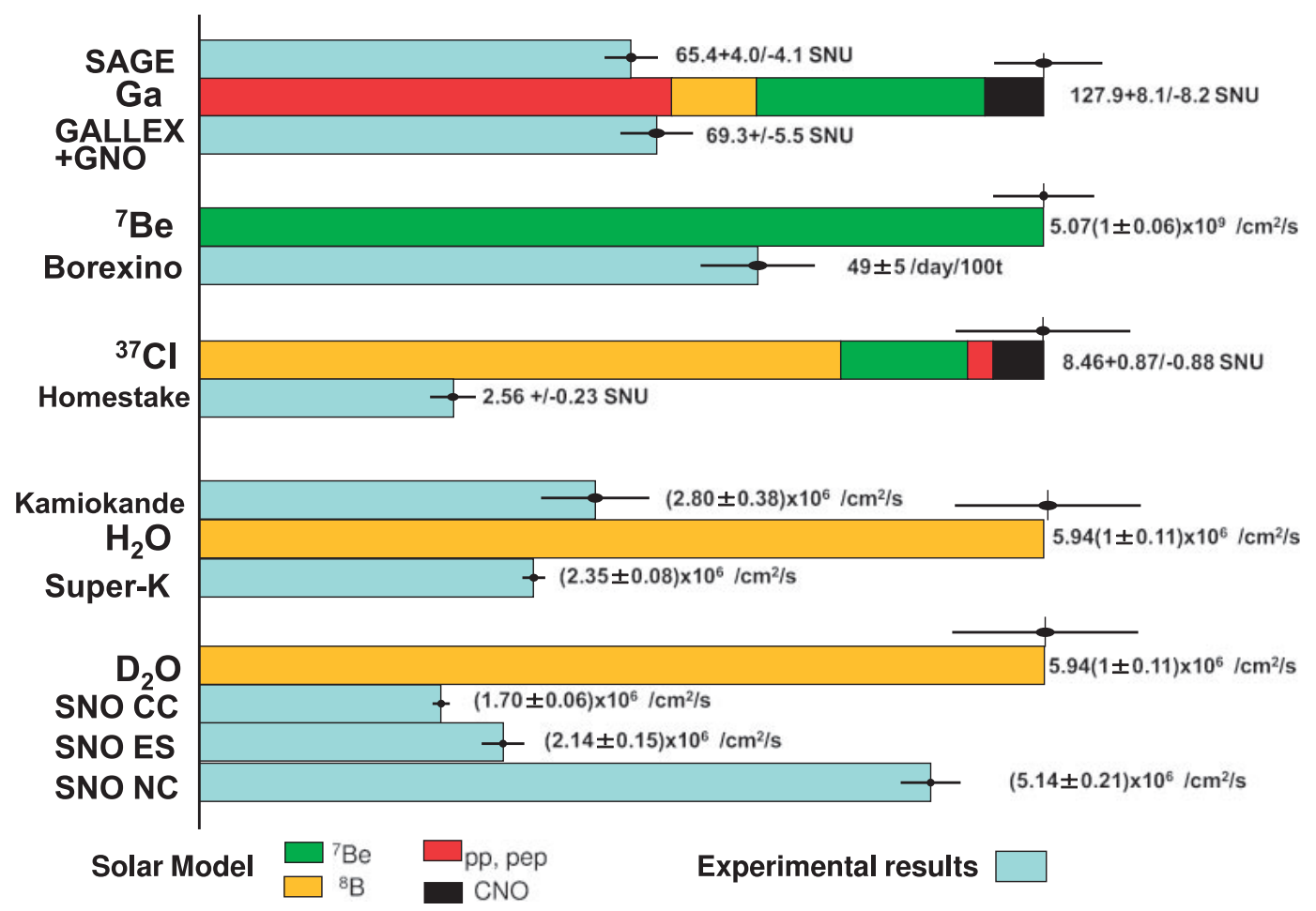

Fig. 16. Results of the solar neutrino experiments (light blue). Measured fluxes are compared with the SSM predictions ${ }^{4)}$ with contributions from neutrino sources of $p p+p e p$ (red), ${ }^{7} \mathrm{Be}$ (green), ${ }^{8} \mathrm{~B}$ (yellow), and $\mathrm{CNO}$ (black).

equation for neutrino oscillation in vacuum can be written as:

$$
i \frac{d}{d t}\left(\begin{array}{c}
\nu_{e} \\
\nu_{X}
\end{array}\right)=\left(\begin{array}{cc}
-\frac{\Delta m^{2}}{4 E} \cos 2 \theta & \frac{\Delta m^{2}}{4 E} \sin 2 \theta \\
\frac{\Delta m^{2}}{4 E} \sin 2 \theta & \frac{\Delta m^{2}}{4 E} \cos 2 \theta
\end{array}\right)\left(\begin{array}{c}
\nu_{e} \\
\nu_{X}
\end{array}\right)
$$

Because of the difference in the forward scattering amplitudes of $\nu_{e}$ and $\nu_{\mu} / \nu_{\tau}$ induced by the presence of electrons in the ambient matter, the Schrödinger equation becomes: $:^{31), 32)}$

$$
\begin{aligned}
i \frac{d}{d t}\left(\begin{array}{c}
\nu_{e} \\
\nu_{X}
\end{array}\right) & \\
= & \left(\begin{array}{cc}
-\frac{\Delta m^{2}}{4 E} \cos 2 \theta+\sqrt{2} G_{F} n_{e} & \frac{\Delta m^{2}}{4 E} \sin 2 \theta \\
\frac{\Delta m^{2}}{4 E} \sin 2 \theta & \frac{\Delta m^{2}}{4 E} \cos 2 \theta
\end{array}\right) \\
& \times\left(\begin{array}{c}
\nu_{e} \\
\nu_{X}
\end{array}\right),
\end{aligned}
$$

where $G_{F}$ is the Fermi coupling constant and $n_{e}$ is the electron number density along the neutrino flight path. For the case of constant matter density, the oscillation can be written using the same formula as Eq. [2] with an effective mixing angle, $\theta_{m}$, defined as

$$
\sin ^{2} 2 \theta_{m}=\frac{\sin ^{2} 2 \theta}{\sin ^{2} 2 \theta+\left(L_{v} / L_{0}-\cos 2 \theta\right)^{2}},
$$

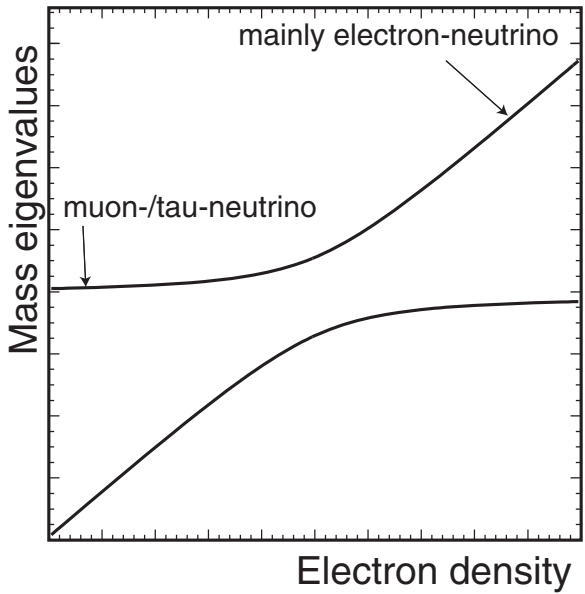

Fig. 17. Mass eigenvalues plotted as a function of electron density for two-neutrino oscillations in matter.

where $L_{v}\left(\equiv \frac{4 \pi E}{\Delta m^{2}}\right)$ is the vacuum oscillation length and $L_{0}$ is

$$
L_{0}=\frac{\sqrt{2} \pi}{G_{F} n_{e}} .
$$

The amplitude of neutrino oscillations is enlarged or suppressed by the matter effect depending on the 


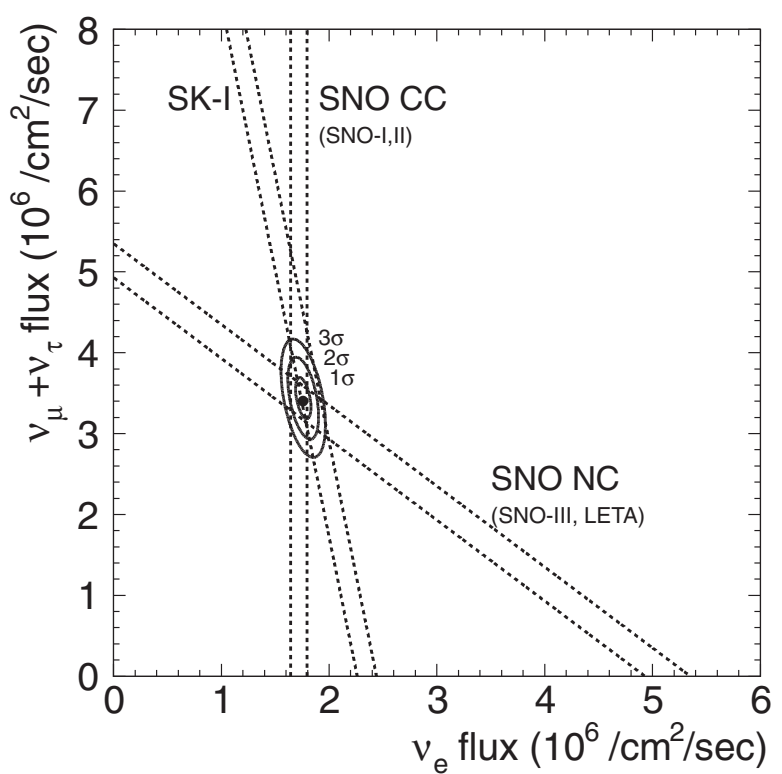

Fig. 18. Flux constraints from measurements by SK-I, SNO CC (SNO-I and II) and SNO NC (SNO-III and LETA). Each dotted band shows the $\pm 1 \sigma$ constraint from each measurement. The circles show $1 \sigma, 2 \sigma$ and $3 \sigma$ constraints obtained by combining the SK and SNO data.

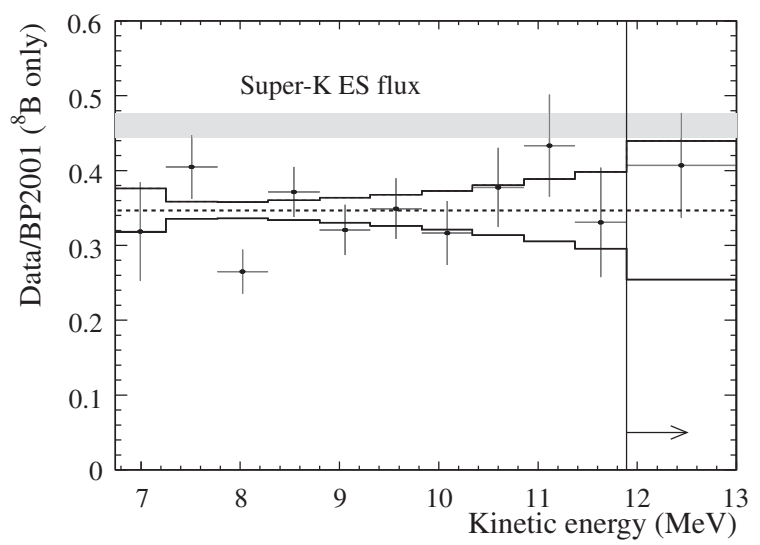

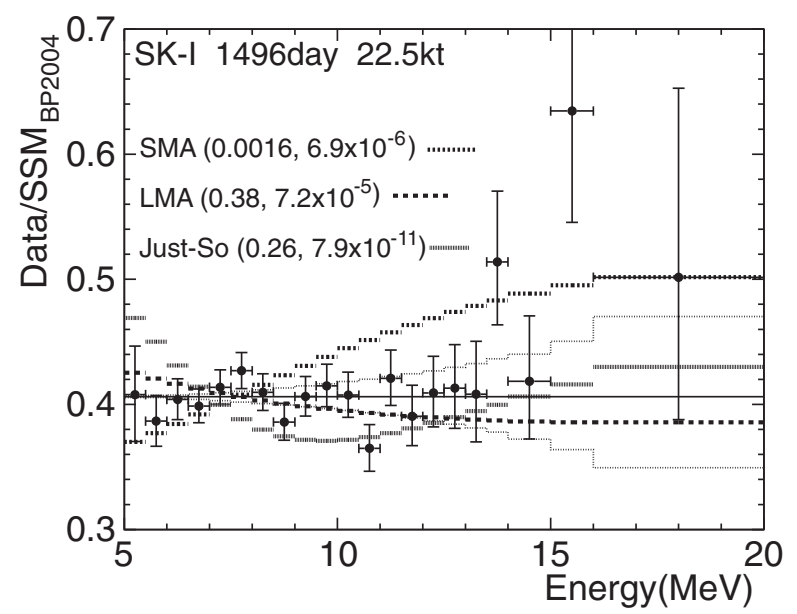

Fig. 19. Measured energy spectrum from SK-I. The horizontal axis is the visible energy of recoil electrons and the vertical axis shows the flux normalized by the SSM prediction without neutrino oscillations. The thick-dotted, -dashed and -fine-dotted histograms show typical energy spectra for SMA, LMA, and Just-so oscillations, respectively.

Fig. 20. Measured energy spectrum from SNO-I ${ }^{26)}$ and SNO-II. ${ }^{27)}$ The left figure shows the observed SNO-I CC electron kinetic energy spectrum normalized by the SSM prediction $\left(\mathrm{BP} 2001^{2)}\right)$ without oscillations. The right figures shows the CC electron kinetic energy spectrum of SNO-II.

oscillation parameters and the ordering of the mass eigenstates. In the matter environment of the sun, the varying matter density can cause an adiabatic transition from $\nu_{e}$ to $\nu_{X}$. This is called the Mikheyev-Smirnov-Wolfenstein (MSW) effect. ${ }^{31), 32)}$ Figure 17 shows the mass eigenvalues plotted as a function of matter density. Electron neutrinos born at the core of the sun, where the matter density is very high, can be transformed into $\nu_{X}$ as they pass

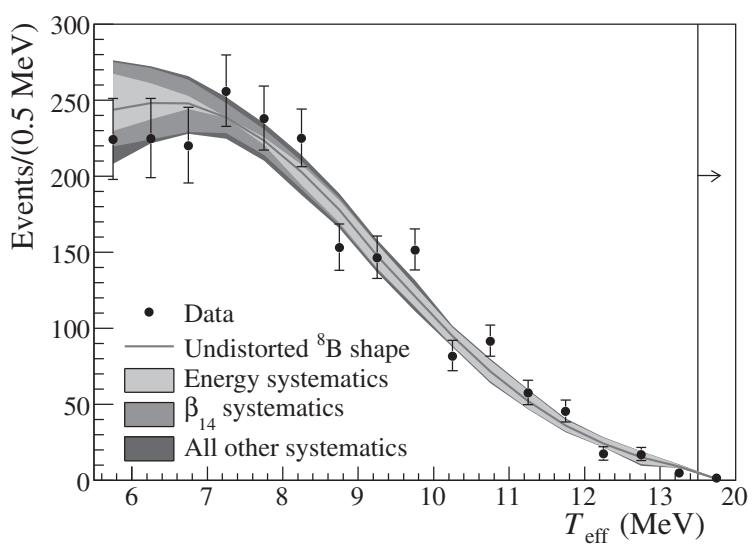

through the sun. Note that $P\left(\nu_{e} \rightarrow \nu_{e}\right)$ cannot be less than $\frac{1}{2}$ unless the oscillation length is close to the distance from the source to the detector (the socalled "just-so" case) as shown in Eq. [3]. However, the matter effect is able to make $P\left(\nu_{e} \rightarrow \nu_{e}\right)$ less than $\frac{1}{2}$ even if the oscillation parameters are not the just-so case.

B. Evidence for solar neutrino oscillation. SK and SNO results are plotted on the plane of $\nu_{e}$ 


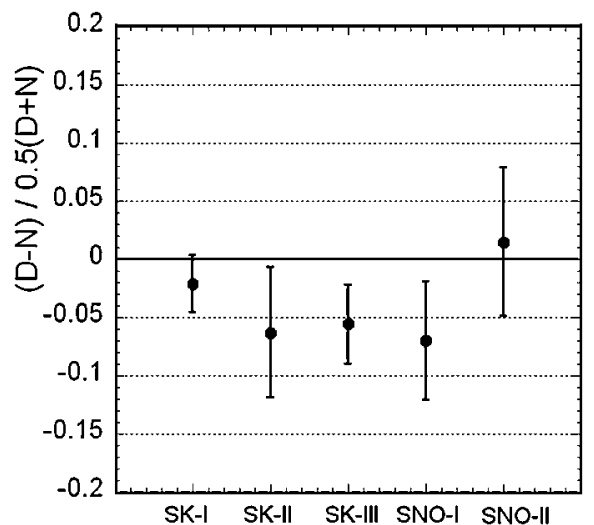

Fig. 21. Summary of day/night asymmetry measurements. Each error is the quadratic sum of the statistical and systematic errors.

flux and $\nu_{\mu}+\nu_{\tau}$ flux in Fig. 18. The slight slope in the SK data in this plot is due to the contribution from $\nu_{\mu}+\nu_{\tau}$ to neutrino-electron scattering. In 2001, a comparison between the SK-I flux and the CC flux measurement of SNO-I gave evidence for a non-zero $\nu_{\mu}+\nu_{\tau}$ flux at $3.3 \sigma$ significance, ${ }^{33)}$ which is direct evidence for solar neutrino oscillations. The statistical significance of the evidence has been greatly improved by the SNO NC measurements (including LETA results) and subsequent further ES measurements from SK-II and SK-III.

C. Energy spectrum and day/night difference. Distortion of the solar neutrino energy spectrum is predicted by MSW oscillations and observations of this distortion can be used to constrain the parameters governing neutrino oscillations.

Figure 19 shows the observed total energy spectrum of SK-I compared with expectations from MSW neutrino oscillations. The observed energy spectrum is almost flat and excludes the small mixing angle (SMA) and just-so solutions, while favoring the large mixing angle (LMA) solution. Figure 20 shows the measured energy spectra from SNO-I CC and SNO-II CC. Both SK and SNO data do not show an energy spectrum distortion yet.

The day/night flux difference is evaluated by an asymmetry parameter $\left(A_{D N}\right)$ defined as $\frac{(d a y-n i g h t)}{\frac{1}{2}(d a y+n i g h t)}$. The asymmetry measured by SK-I, ${ }^{15)}$ SK-II ${ }^{17)}$ and

$$
\begin{aligned}
\text { SK-III } & \\
& \\
A_{D N}^{S K-I} & =-0.021 \pm 0.020 \text { (stat. })_{-0.012}^{+0.013} \text { (sys.) } \\
A_{D N}^{S K-I I} & =-0.063 \pm 0.042(\text { stat. }) \pm 0.037 \text { (sys.) } \\
A_{D N}^{S K-I I I} & =-0.056 \pm 0.031 \text { (stat.) } \pm 0.013 \text { (sys.) }
\end{aligned}
$$

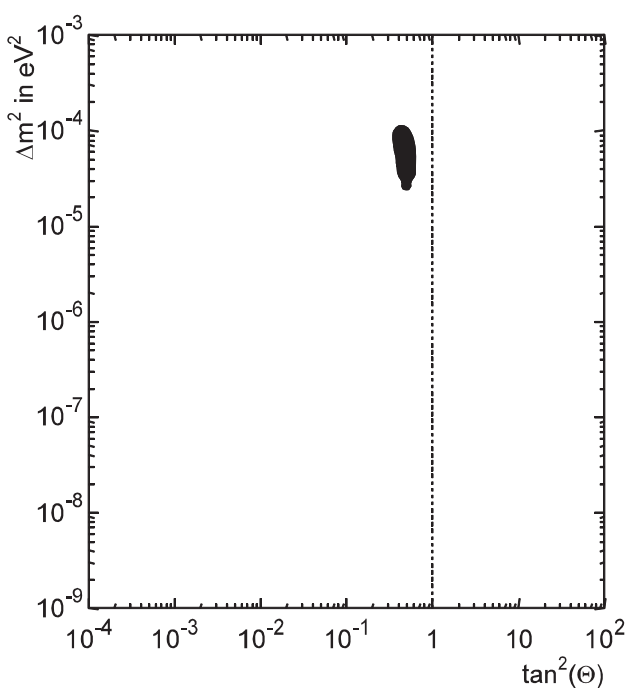

Fig. 22. Allowed region from SK-I, II, III combined analysis. The ${ }^{8} \mathrm{~B}$ flux is constrained by SNO NC rate (LITA and phase-III).

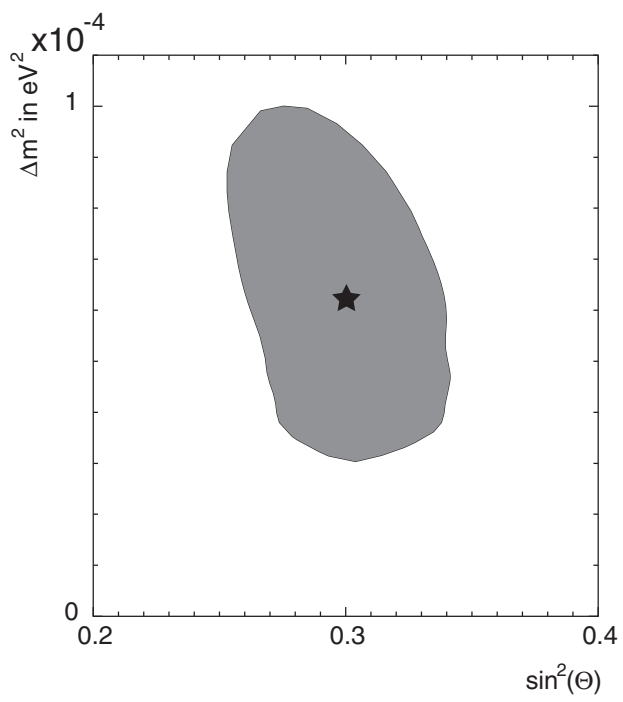

Fig. 23. The allowed region of oscillation parameters for all solar neutrino experiments with $95 \%$ C.L.

SNO determined day/night asymmetries for CC and NC channels separately. With a constraint of $A_{D N}=0$ for the NC channel, the measured day/night asymmetries by SNO-I and SNO-II ${ }^{27)}$ are

$$
\begin{aligned}
& \left.A_{D N}^{S N O-I, C C}=-0.07 \pm 0.049 \text { (stat. }\right)_{-0.013}^{+0.012} \text { (sys.) } \\
& A_{D N}^{S N O-I I, C C}=0.015 \pm 0.058 \text { (stat.) } \pm 0.027 \text { (sys.). }
\end{aligned}
$$

A summary of these measurements is shown in Fig. 21. Because of the contribution of $\nu_{\mu}$ and $\nu_{\tau}$ in the SK electron scattering data, the expected $A_{D N}$ 


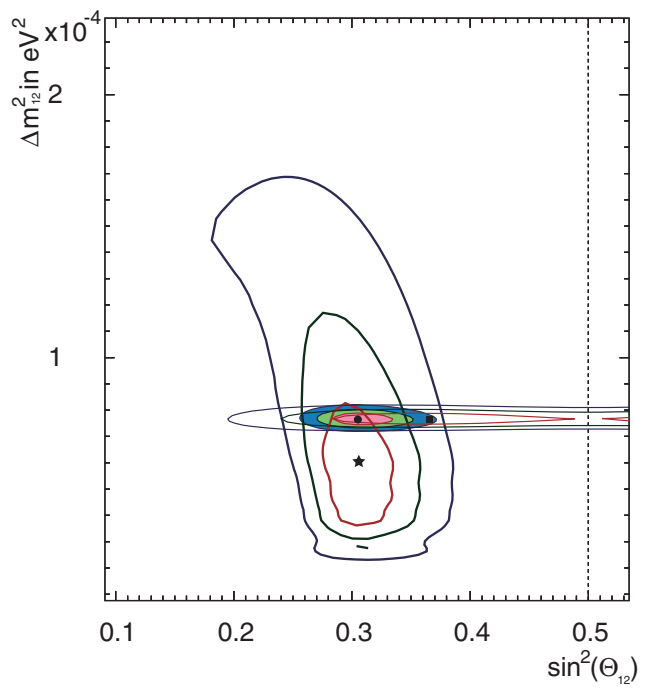

Fig. 24. Allowed region of oscillation parameters of $\theta_{12}$ and $\Delta m_{12}^{2}$ obtained by the three-flavor solar analysis (thick lines). The thin lines show the allowed regions from the KamLAND reactor neutrino analysis. The filled areas show the combined solar and reactor analysis. For all regions, the innermost area (red), the middle area (green) and the outermost area (blue) show the 68.3, $95,99.7 \%$ C.L. respectively.

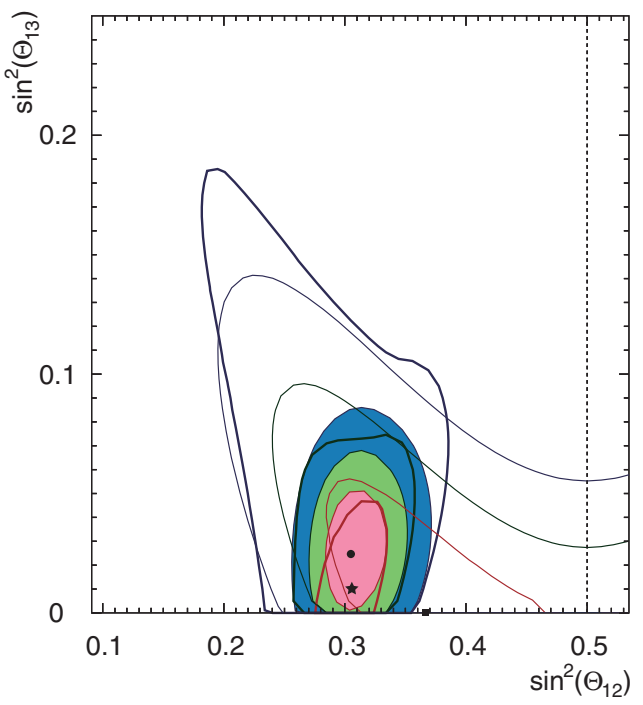

Fig. 25. Allowed region of oscillation parameters of $\theta_{12}$ and $\theta_{13}$ obtained by the three-flavor solar analysis (thick lines). The thin lines show the allowed regions from the KamLAND reactor neutrino analysis. The filled areas show the combined solar and reactor analysis. For all regions, the innermost area (red), the middle area (green) and the outermost area (blue) show the 68.3, $95,99.7 \%$ C.L. respectively.

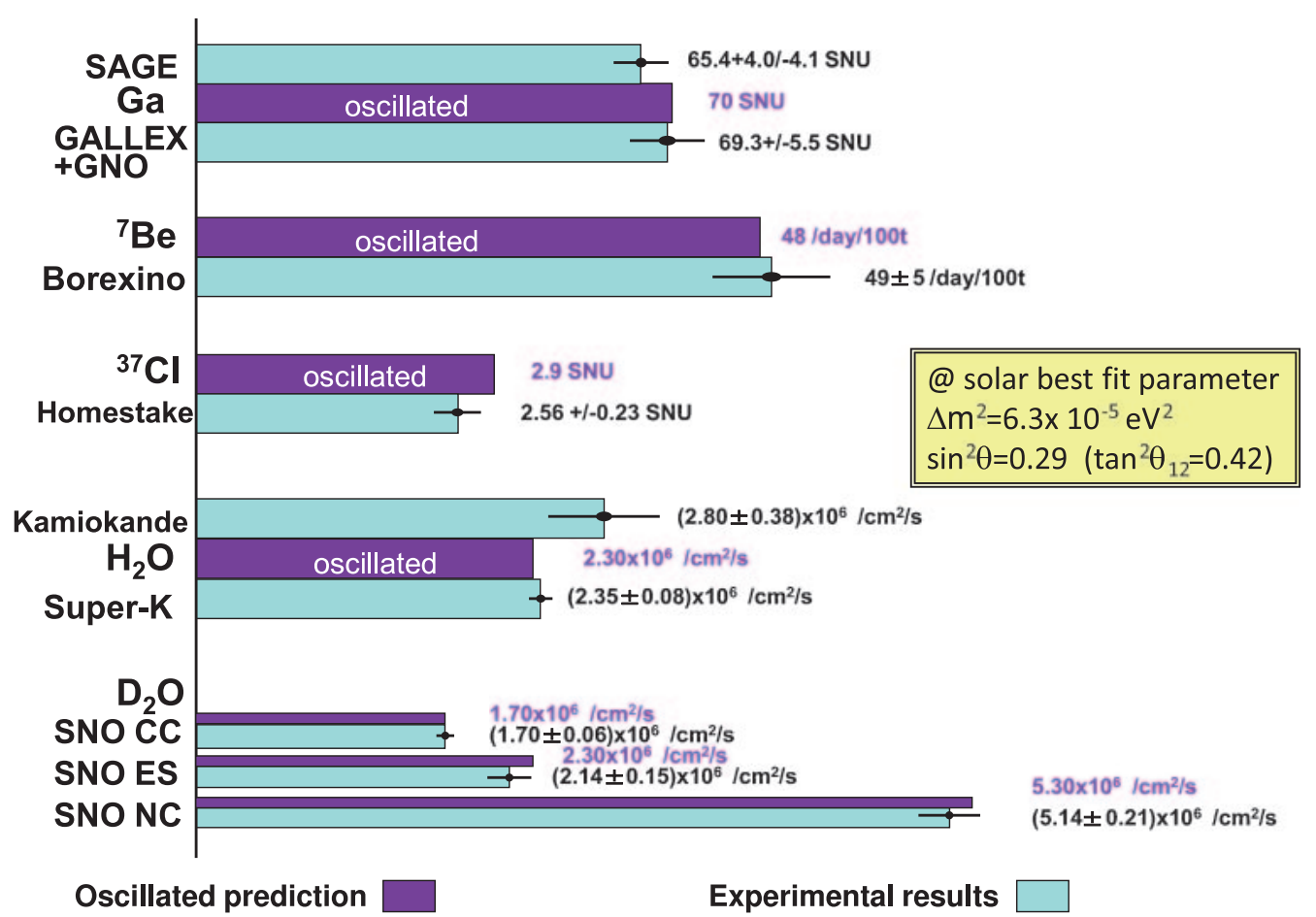

Fig. 26. Results from the solar neutrino experiments (light blue) compared with the SSM prediction including the effect of neutrino oscillations. 
of SNO CC is about $50 \%$ larger than $A_{D N}$ of SK for typical LMA parameters. SK and SNO have not seen a definite finite value of the asymmetry yet, but the current values are consistent with the expectation. Note that the expected $A_{D N}$ value of SK is about $1.5 \%$ for the best fit oscillation parameter described in the next section.

D. Oscillation parameters. Neutrino oscillation parameters obtained by the solar neutrino measurements are described in this subsection. Figure 22 shows the allowed region of neutrino oscillation parameters obtained by the SK-I, II, III combined analysis in which fluxes, energy spectrums, and the day/night asymmetry of SK data are taken into account. The ${ }^{8} \mathrm{~B}$ flux is constrained by the SNO NC rate (LETA and phaseIII). A unique solution in the LMA region is obtained by the SK analysis constrained to the SNO NC flux.

Allowed regions for the solar oscillation parameters obtained by combining the results of all solar neutrino experiments (chlorine, gallium, SK, SNO, and Borexino) is shown in Fig. 23. The best-fit oscillation parameters are obtained to be $\sin ^{2} \theta=$ $0.30_{-0.01}^{+0.02}\left(\tan ^{2} \theta=0.42_{-0.02}^{+0.04}\right)$ and $\Delta m^{2}=6.2_{-1.9}^{+1.1} \times$ $10^{-5} \mathrm{eV}^{2}$.

E. Three flavor oscillation analysis. The oscillation analysis described in the previous section assumed two flavor neutrinos expressed by Eq. [1]. In this section, a recent analysis taking into account all three flavors is described.

The relation between the mass and interaction eigenstates is described by

$$
\left(\begin{array}{c}
\nu_{\mathrm{e}} \\
\nu_{\mu} \\
\nu_{\tau}
\end{array}\right)=\left(\begin{array}{lll}
U_{e 1} & U_{e 2} & U_{e 3} \\
U_{\mu 1} & U_{\mu 2} & U_{\mu 3} \\
U_{\tau 1} & U_{\tau 2} & U_{\tau 3}
\end{array}\right)\left(\begin{array}{c}
\nu_{1} \\
\nu_{2} \\
\nu_{3}
\end{array}\right)
$$

The unitary matrix $U$ is the Maki-NakagawaSakata-Pontecorvo (MNSP) matrix and it can be decomposed into three angles and a phase:

$$
\begin{aligned}
U & =\left(\begin{array}{ccc}
1 & 0 & 0 \\
0 & c_{23} & s_{23} \\
0 & -s_{23} & c_{23}
\end{array}\right)\left(\begin{array}{ccc}
c_{13} & 0 & s_{13} e^{i \delta} \\
0 & 1 & 0 \\
-s_{13} e^{-i \delta} & 0 & c_{13}
\end{array}\right)\left(\begin{array}{ccc}
c_{21} & s_{12} & 0 \\
-s_{12} & c_{12} & 0 \\
0 & 0 & 1
\end{array}\right) \\
& =\left(\begin{array}{ccc}
c_{12} c_{13} & s_{12} c_{13} & s_{13} e^{-i \delta} \\
-s_{12} c_{23}-c_{12} s_{23} s_{13} e^{i \delta} & c_{12} c_{23}-s_{12} s_{23} s_{13} e^{i \delta} & s_{23} c_{13} \\
s_{12} s_{23}-c_{12} c_{23} s_{13} e^{i \delta} & -c_{12} s_{23}-s_{12} c_{23} s_{13} e^{i \delta} & c_{23} c_{13}
\end{array}\right),
\end{aligned}
$$

where $c_{i j} \equiv \cos \theta_{i j}$ and $s_{i j} \equiv \sin \theta_{i j}$. In the solar neutrino oscillation analysis, the oscillation probability can be calculated using three parameters, $\theta_{12}$, $\theta_{13}$, and $\Delta m_{12}^{2}$, because $\Delta m_{12}^{2} \ll \Delta m_{23}^{2} \sim \Delta m_{13}^{2}$. Figure 24 shows the allowed region of the oscillation parameters, $\left(\theta_{12}, \Delta m_{12}^{2}\right)$, obtained by the three-flavor global analysis. In this plot, the $\theta_{13}$ parameter is varied to minimize the $\chi^{2}$ at each point in the plot. Figure 24 also shows the contour obtained by the KamLAND reactor neutrino data ${ }^{34)}$ (details of this analyses are described in ref. 18). Figure 25 shows the allowed mixing angles, $\theta_{12}$ and $\theta_{13}$ for the global solar analysis, KamLAND reactor and their combined analysis. In the global solar analysis larger values of $\theta_{13}$ prefer the larger values of $\theta_{12}$, while in the KamLAND reactor contour the larger value of $\theta_{13}$ prefers the smaller value of $\theta_{12}$. The best fit value of $\sin ^{2} \theta_{13}$ is 0.01 , and an upper bound is obtained $\sin ^{2} \theta_{13}$ at the $95 \%$ C.L. for the global solar analysis. Combining with the KamLAND contour, the best fit value of $\sin ^{2} \theta_{13}$ is $0.025_{-0.016}^{+0.018}$ and the $95 \%$ C.L. upper limit is $\sin ^{2} \theta_{13}<0.059$.

F. Summary and future prospects. Precise measurement of solar neutrinos by SK and SNO indicate that solar neutrino problem is due to neutrino oscillations. Figure 26 shows a comparison between the measured neutrino fluxes and the SSM prediction taking into account the effect of neutrino oscillations. The measured fluxes are consistent with the SSM prediction with neutrino oscillations. The obtained oscillation parameters are consistent with those obtained by the reactor neutrino analysis of KamLAND. So, the longstanding solar neutrino problem has been solved.

Is it the end of the story? I do not think so. We have not yet observed the smoking gun features of solar neutrino oscillations, such as the distortion of the energy spectrum and the difference between the day and night fluxes. Unfortunately these effects are small and we need more solar neutrino data to observe them. As shown in Table 1, recent solar models have a problem related to the solar composition. The model which uses the most precise composition measurements cannot reproduce the depth of the convective zone and the helium abundance. Further solar neutrino measurements, including CNO neutrino measurements, should contribute to solve this new solar problem.

The most abundant solar neutrino source is $p p$ neutrinos and the SSM predicts its flux with an accuracy of $\sim 1 \%$. However, it is measured only by the gallium experiments as a part of their counting rate. Real time $p p$ solar neutrino measurements are needed in future. ${ }^{35)}$ 


\section{References}

1) Bethe, H.A. (1939) Energy production in stars. Phys. Rev. 55, 434-456.

2) Bahcall, J.N., Pinsonneault, M.H. and Basu, S. (2001) Solar models: Current epoch and time dependences, neutrinos, and helioseismological properties. Astrophys. J. 555, 990-1012.

3) Bahcall, J.N. and Pinsonneault, M.H. (2004) What do we (not) know theoretically about solar neutrino fluxes? Phys. Rev. Lett. 92, 121301.

4) Pena-Garay, C. and Serenelli, A. (2008) Solar neutrinos and the solar composition problem. arXiv:0811.2424 [astro-ph].

5) Serenelli, A., Haxton, W. and Pena-Garay, C. (2010) Solar models with accretion. I. Application to the solar abundance problem. arXiv:1104.1639 [astro-ph].

6) Adelberger, E.G. et al. (2010) Solar fusion cross sections II: the pp chain and CNO cycles. arXiv:1004.2318 [nucl-ex].

7) Grevesse, N. and Sauval, A.J. (1998) Standard solar composition. Space Sci. Rev. 85, 161-174.

8) Asplund, M., Grevesse, N., Sauval, A.J. and Scott, P. (2009) The chemical composition of the Sun. Annu. Rev. Astron. Astrophys. 47, 481-522.

9) Cleveland, B.T. et al. (1998) Measurement of the solar electron neutrino flux with the Homestake chlorine detector. Astrophys. J. 496, 505-526.

10) Hirata, K.S. et al. (Kamiokande-II Collaboration) (1989) Observation of ${ }^{8} \mathrm{~B}$ solar neutrinos in the Kamiokande-II Detector. Phys. Rev. Lett. 63, 16-19.

11) Fukuda, Y. et al. (Kamiokande Collaboration) (1996) Solar neutrino data covering solar cycle 22. Phys. Rev. Lett. 77, 1683-1686.

12) Nakahata, M. et al. (Super-Kamiokande Collaboration) (1999) Calibration of Super-Kamiokande using an electron LINAC. Nucl. Instrum. Methods Phys. Res. A 421, 113-129.

13) Blaufuss, E. et al. (Super-Kamiokande Collaboration) (2001) ${ }^{16} \mathrm{~N}$ as a calibration source for SuperKamiokande. Nucl. Instrum. Methods Phys. Res. A 458, 638-649.

14) Fukuda, S. et al. (Super-Kamiokande Collaboration) (2001) Solar ${ }^{8} \mathrm{~B}$ and hep neutrino measurements from 1258 days of Super-Kamiokande data. Phys. Rev. Lett. 86, 5651-5655.

15) Fukuda, S. et al. (Super-Kamiokande Collaboration) (2002) Determination of solar neutrino oscillation parameters using 1496 days of Super-Kamiokande I data. Phys. Lett. B 539, 179-187.

16) Hosaka, J. et al. (Super-Kamiokande Collaboration) (2006) Solar neutrino measurements in superKamiokande-I. Phys. Rev. D 73, 112001.

17) Cravens, J.P. et al. (Super-Kamiokande Collaboration) (2008) Solar neutrino measurements in Super-Kamiokande-II. Phys. Rev. D 78, 032002.

18) Abe, K. et al. (Super-Kamiokande Collaboration) (2010) Solar neutrino results in SuperKamiokande-III. Phys. Rev. D 83, 052010.

19) Abdurashitov, J.N. et al. (SAGE Collaboration) (1994) Results from SAGE. Phys. Lett. B 328,
$234-248$.

20) Gavrin, V.N. et al. (SAGE Collaboration) (2001) Solar neutrino results from SAGE. Nucl. Phys. B (Proc. Suppl.) 91, 36-43.

21) Abdurashitov, J.N. et al. (SAGE Collaboration) (2009) Measurement of the solar neutrino capture rate with gallium metal. III: Results for the $2002-$ 2007 data-taking period. Phys. Rev. C 80, 015807.

22) Anselmann, P. et al. (GALLEX Collaboration) (1994) GALLEX results from the first 30 solar neutrino runs. Phys. Lett. B 327, 377-385.

23) Anselmann, P. et al. (GALLEX Collaboration) (1995) GALLEX solar neutrino observations: Complete results for GALLEX II. Phys. Lett. B 357, 237-247.

24) Hampel, W. et al. (GALLEX Collaboration) (1996) GALLEX solar neutrino observations: Results for GALLEX III. Phys. Lett. B 388, 384-396.

25) Altmann, M. et al. (GNO Collaboration) (2005) Complete results for five years of GNO solar neutrino observations. Phys. Lett. B 616, 174-190.

26) Ahmad, Q.R. et al. (SNO Collaboration) (2002) Direct evidence for neutrino flavor transformation from neutral current interactions in the Sudbury Neutrino Observatory. Phys. Rev. Lett. 89, 011301.

27) Aharmim, B. et al. (SNO Collaboration) (2005) Electron energy spectra, fluxes, and day-night asymmetries of ${ }^{8} \mathrm{~B}$ solar neutrinos from measurements with $\mathrm{NaCl}$ dissolved in the heavy-water detector at the Sudbury Neutrino Observatory. Phys. Rev. C 72, 055502.

28) Aharmim, B. et al. (SNO Collaboration) (2008) Using an array of ${ }^{3} \mathrm{He}$ proportional counters at the Sudbury Neutrino Observatory. Phys. Rev. Lett. 101, 111301.

29) Aharmim, B. et al. (SNO Collaboration) (2010) Low energy threshold analysis of the phase I and phase II data sets of the Sudbury Neutrino Observatory. Phys. Rev. C 81, 055504.

30) Arpesella, C. et al. (Borexino Collaboration) (2008) Direct measurement of the ${ }^{7} \mathrm{Be}$ solar neutrino flux with 192 days of Borexino data. Phys. Rev. Lett. 101, 091302.

31) Mikheyev, S.P. and Smirnov, A.Y. (1985) Resonance amplification of oscillations in matter and spectroscopy of solar neutrinos. Sov. Jour. Nucl. Phys. 42, 913-917.

32) Wolfenstein, L. (1978) Neutrino oscillations in matter. Phys. Rev. D 17, 2369-2374.

33) Ahmad, Q.R. et al. (SNO Collaboration) (2001) Measurement of the rate of $\nu_{e}+d \rightarrow p+p+e^{-}$ interactions produced by ${ }^{8} \mathrm{~B}$ solar neutrinos at the Sudbury Neutrino Observatory. Phys. Rev. Lett. 87, 071301.

34) Abe, S. et al. (KamLAND Collaboration) (2008) Precision measurement of neutrino oscillation parameters with KamLAND. Phys. Rev. Lett. 100, 221803.

35) Nakahata, M. (2005) Future solar neutrino experiments. Nucl. Phys. (Proc. Suppl.) 145, 23-28.

(Received Jan. 15, 2011; accepted Mar. 22, 2011) 


\section{Profile}

Masayuki Nakahata was born in 1959. In 1982, he started his scientific career at the graduate course in the University of Tokyo under the supervision of Professor Masatoshi Koshiba. He joined the Kamiokande experiment from its construction stage. He analyzed the supernova data of Kamiokande in 1987. He received Ph.D. in physics from University of Tokyo in 1988 with the solar neutrino analysis of Kamiokande. In 1988, he got a position in the Institute for Cosmic Ray Research (ICRR), University of Tokyo, and continued the solar neutrino analysis of Kamiokande. During this time, he observed solar neutrino signals by the realtime detector for the first time in the world. From 1990 to 1993, he was moved to Hamburg university as a postdoc, and performed a research at the electron-proton collider HERA as a member of the ZEUS experiment. In 1993, after he

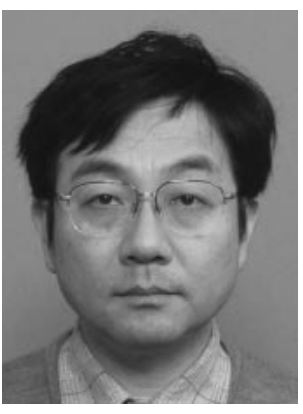
came back to Japan, he was moved to Kamioka for the construction of the Super-Kamiokande detector. Since then, he has been working for Super-Kamiokande, especially for low energy neutrino physics. For the studies of solar neutrinos and neutrino oscillations, he received the 1989 Inoue Research Award for Young Scientists, the 2001 Nishina Memorial Prize (with Yoichiro Suzuki), the 2009 Inoue Prize for Science, the 2011 Yoji Totsuka Prize. 